\title{
Programas de promocão de atividade física envolvendo camínhada nas universidades públicas brasileiras
}

\section{Promotion of physical activity programs involving walking in Brazilian public universities}

Natália Oiring de Castro Cezar

Mariana Luciano Almeida

Giovana de Oliveira Padula

Agatha Beatriz Wolf Cassavia

Erica Nestor Souza

José Marques Novo Jr.

Grace Angélica de Oliveira Gomes

\section{RESUMO}

Diante da necessidade de ampliar o conhecimento de pesquisadores e universidades sobre a existência de programas de caminhada e de atividade física envolvendo caminhada, desenvolvidos em universidades públicas brasileiras, este estudo teve o objetivo de identificar tais projetos e programas encontrados neste perfil, bem como as publicações referentes a estes. O critério de inclusão constituiu-se de programas implantados em instituições públicas, desenvolvidos entre 1984 e 2013 e artigos relacionados publicados nas bases de dados SciELO, LILACS e Google Acadêmico. Abordaram-se universidades públicas brasileiras, totalizando 99 instituições. Foram encontrados 103 programas que envolvem caminhada, distribuídos em 54 universidades públicas brasileiras. Os estados com maior número de programas são: São Paulo (SP), totalizando 19 programas concentrados em cinco universidades, Minas Gerais, com 15 programas entre oito universidades e Rio Grande do Sul (RS) e Santa Catarina (SC), com 11 e 12, respectivamente, desenvolvidos em sete universidades. A maioria dos programas encontrados tem como público alvo os idosos, visando a promoção da saúde. Das 54 universidades que oferecem programa, 30 realizaram 339 publicações científicas sobre os programas desenvolvidos, destaque para SP, SC e RS. Em conclusão, encontramos que mais da metade das universidades brasileiras possuem programas de atividade física envolvendo caminhada ou de caminhada e há um número considerável de publicações relacionadas. A continuidade e expansão desses programas são de grande importância já que se trata de uma alternativa simples de promoção da saúde, de baixo custo e que apresenta melhorias consideravelmente relevantes para a qualidade de vida de quem adere ao programa.

\section{PALAVRAS-CHAVE}

Exercícios físicos; Promoção da saúde; Revisão; Universidades; Avaliação de programas; Projetos de saúde.

\begin{abstract}
Faced with the need to expand the knowledge about the existence of physical activity programs of walking which programs which involving walking developed in Brazilian public universities, the present study aimed to describe projects found in this profile to render knowledge of researchers and universities the existence and importance of projects and programs aimed at physical activity. The inclusion criteria consisted of programs implemented in public institutions, developed between 1984 and 2013 and related articles published in the SciELO, LILACS and Google Scholar database. Were interviewed 99 public universities. Were found 103 programs involving walking, distributed in 54 Brazilian public universities. The states with the highest number of programs are: Sao Paulo (SP), totaling 19 programs concentrated in five universities, Minas Gerais, with 15 programs in eight universities and Rio Grande do Sul (RS), and Santa Catarina (SC), 11 and 12, respectively, developed in seven universities. The programs found are mostly for the elderly audience, with the goal of promoting health. Of the 54 universities that offer the program, 30 of them performed 339 scientific publications about the programs developed, especially SP, SC and RS. In conclusion, we found that more than half of Brazilian universities have physical activity programs of walking and involving walking. The continuation and expansion of these programs are of great importance since these are a simple alternative for health promotion, low cost and that has pretty significant improvements to the quality of life of those who adhere to the program.
\end{abstract}

\section{KEYWORDS}

Exercise; Health Promotion; Review; Universities; Program Evaluation
Rev Bras Ativ Fis Saúde p. 441-454 DOI

http://dx.doi.org/10.12820/rbafs.v.19n4p441

Universidade Federal de São Carlos - UFSCar. 


\section{INTRODUCCÃO}

A inatividade física vem sendo considerada um fator de risco primário e independente para muitos agravos à saúde, particularmente às doenças cardiovasculares e metabólicas ${ }^{1}$. Desde meados dos anos 90 as pesquisas vêm apoiando a prática de qualquer tipo de atividade física que trouxesse benefícios para saúde e a capacidade funcional, que não cause danos ou riscos ${ }^{2}$. Embora a literatura já tenha acumulado evidências que comprovem os benefícios da atividade física regular para a saúde, estudos têm apontado baixos níveis desse comportamento na população ${ }^{3-4}$. Considerando apenas a atividade física no lazer, um levantamento do Ministério da Saúde indica que, entre os adultos das capitais brasileiras, a frequência da prática de atividade física no lazer é de $33,5 \%$, sendo mais frequente no sexo masculino (41,5\%) do que no sexo feminino $(26,5 \%)^{5}$.

Diante do exposto, evidencia-se o aumento do risco de desenvolvimento de diversas Doenças Crônicas Não Transmissíveis (DCNT), tendo maior prevalência na população idosa ${ }^{6}$. Essas doenças repercutem na situação econômica do país, e podem gerar absenteísmo no trabalho, incapacidades permanentes e altos custos com tratamento e reabilitação ${ }^{7}$. Considerando os altos custos em saúde gerados pelas complicações dessas doenças, é possível identificar que o nosso país precisa investir mais na prevenção e controle das DCNT, por meio da promoção da atividade física, para que os custos sejam diminuídos ${ }^{8}$.

Nesse sentido, evidencia-se a importância de garantir aos adultos e idosos, não somente uma sobrevida maior, mas melhores condições de vida. Como uma opção importante de prevenção e tratamento dessas patologias de alta prevalência em adultos e idosos, muitas pesquisas têm demonstrado que quem pratica regularmente atividade física apresenta um risco diminuído para o desenvolvimento de várias doenças 9 . Apesar do empenho internacional em incluir a atividade física na agenda de ações de saúde, há evidências de que as escassas iniciativas têm sido bem sucedidas na população que é submetida à prática de atividade física ${ }^{10}$.

Uma das atividades físicas mais praticadas pela população é a caminhada, que deve ser recomendada por ser um movimento natural, de fácil realização e de ótimos resultados, para a saúde e o condicionamento físico ${ }^{3,11}$. A caminhada não necessita de equipamentos especiais para a sua prática, além de ser considerada uma das atividades físicas mais seguras. Do ponto de vista cardiovascular e ortopédico, ela apresenta um alto índice de aderência em exercícios para a promoção de saúde e prevenção de doenças ${ }^{12}$.

Políticas públicas de promoção da saúde têm sido divulgadas enfatizando a prática de caminhadas como medida preventiva e de controle das DCNT, aconselhando a população ao exercício aeróbico regular ${ }^{13-14}$.

Um dos contextos em que a promoção de atividade física é realizada é a extensão universitária, que em alguns casos une-se ao ensino e à pesquisa; a qual tem como interesse produzir conhecimento científico a partir da vivência prática aliada à promoção de qualidade de vida para os participantes dos projetos. A extensão na universidade pública compõe-se de um conjunto de ações pertinentes à sua missão institucional de mediação entre as práticas acadêmicas e as demandas da sociedade, de modo a permitir o diálogo entre a sabedoria popular e a científica ${ }^{15}$. 
Nesse sentido foram criados no Brasil, dentro das universidades públicas, programas e projetos que promovem a prática regular de atividade física. Muitos desses programas possuem anos de existência e tem sido utilizados como exemplos bem sucedidos para outras universidades na implementação de programas de atividade física ${ }^{11,16,17,18}$.

Entretanto, existe a necessidade de sistematização sobre a existência desses programas por meio de uma descrição que abordem a respeito de programas de atividade física implantados em universidades. O conhecimento desses programas pode colaborar como forma de exemplo positivo para gestores e docentes de outras universidades e/ou prefeituras que têm o intuito de apoiar programas de atividade física orientada em seus respectivos municípios. Gestores e docentes podem obter informações e orientações sobre a implantação e funcionamento de programas de tal porte com universidades que já o fizeram. Além disso, o conhecimento sobre o número de publicações advindos da vivência prática desses projetos pode indicar o quanto os pesquisadores brasileiros tem divulgado no meio científico a aplicação de ações preconizadas nas políticas públicas de promoção da saúde.

Universidades privadas também promovem programas e projetos de atividade física que envolvem caminhada, porém neste contexto os projetos de extensão e pesquisa não são tão frequentes quanto nas universidades públicas. Além disso, existe a instabilidade dos cursos de graduação em função da estabilidade financeira das instituições privadas.

Nesta mesma direção, outro ponto relevante é a importância de mostrar como o ensino superior público tem desenvolvido os âmbitos de extensão e pesquisa na área de atividade física, especialmente em relação à prática de caminhada. Dessa forma, justifica-se o foco do estudo nas universidades públicas, justamente por conta do comprometimento que estas tem com o fortalecimento da função da Universidade, isto é, produzir, sistematizar e difundir conhecimento, desenvolvendo suas atividades de pesquisa e ensino interligadas com as demandas dos setores externos (vários segmentos da população) por meio de ações de extensão que, muitas vezes não estão presentes nas instituições privadas nesse nível de estrutura acadêmica.

Diante do exposto o presente estudo tem como objetivo descrever os projetos e programas de atividade física orientada envolvendo caminhada em universidades brasileiras e suas respectivas publicações.

\section{MÉTODOS}

Trata-se de um estudo descritivo realizado entre abril e novembro de 2013, com um primeiro objetivo relacionado à prospecção para identificação dos programas de caminhada e que envolvam caminhada, sejam atuais ou antigos, em universidades e, um segundo, relacionado à busca dos artigos científicos referentes a esses programas.

Primeiramente, foram definidos os critérios de inclusão, os quais se referem a programas implantados em universidades federais e estaduais entre 1984 e 2013, vigentes e encerrados, atendendo aos termos e descritores "programa", "projeto", “caminhada”, "atividade física/motora”, "saúde”, "universidade federal" e "universidade estadual”, physical activity, healthy, guided walk, federal university, program, project, state university, walk healthy. Os dados foram 
pesquisados nas bases de dados SciELO, LILACS e Google Acadêmico, nos sites dos programas de caminhada orientada das universidades e no currículo lattes dos coordenadores. $\mathrm{O}$ critério de exclusão foi a impossibilidade de contatar a Universidade ou suas respectivas informações. Segue abaixo as etapas realizadas para a busca de programas e de artigos científicos relacionados.

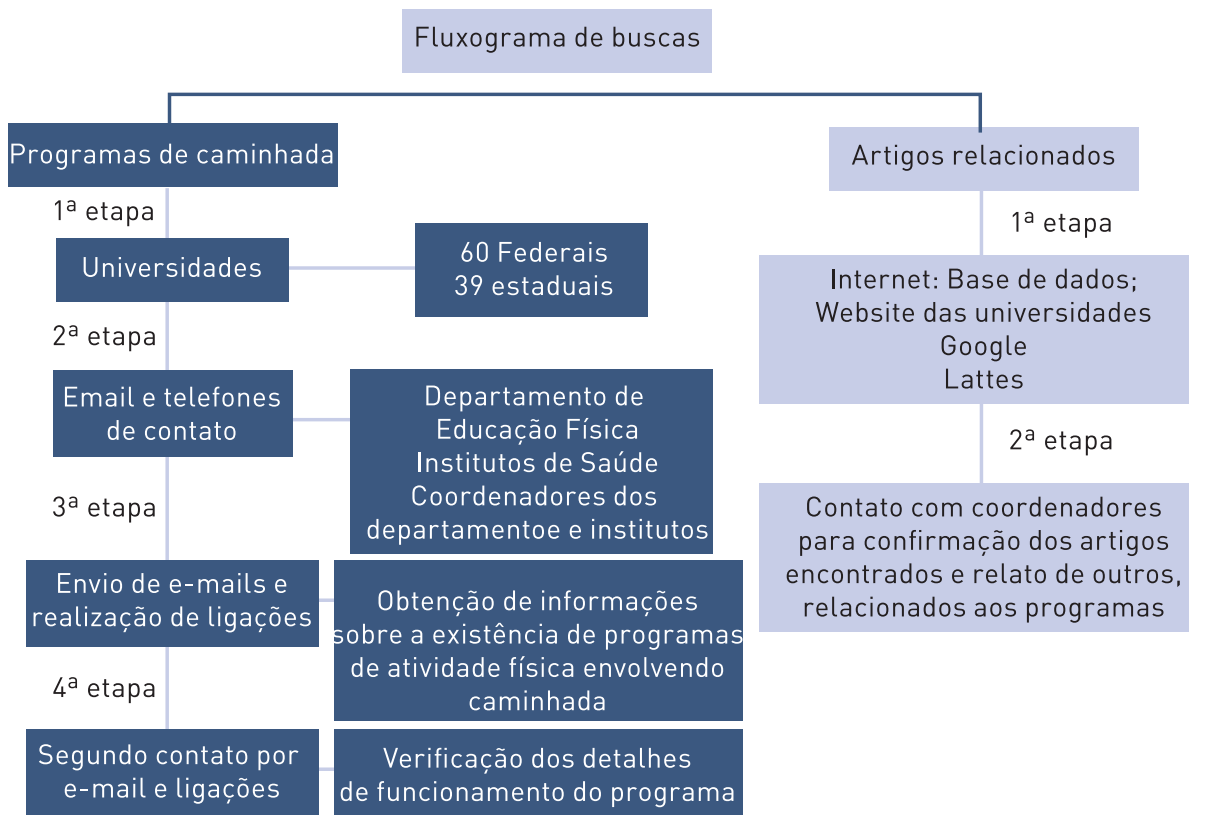

FIGURA 1 - fluxograma da metodologia utilizada na pesquisa para encontrar os programas e os artigos relacionados aos programas

O método utilizado para a obtenção dos dados presentes na pesquisa no que se refere à existência dos programas e projetos se baseou em cinco etapas. Inicialmente foi feita a busca das universidades alvo - todas as universidades públicas brasileiras - sendo 60 federais e 39 estaduais. Posteriormente, a segunda etapa se referiu à busca de e-mails e telefones da Pró-reitoria de Extensão (PROEX), do Departamento de Educação Física (DEF) ou similares, e dos coordenadores dos programas. A terceira etapa foi o envio de e-mails, bem como realização de ligações a todas as PROEX e DEF, a fim de obter informações de possíveis Programas de atividade física e quais desses envolviam caminhada. A quarta etapa acena em um segundo envio de e-mails e realização de ligações telefônicas com o intuito de contatar diretamente os coordenadores dos programas e projetos existentes a fim de obter os dados das Universidades que ainda não haviam retornado o contato e conseguir mais informações por meio de um roteiro elaborado sobre o número de integrantes, o público alvo, ano de implantação, quantas vezes ocorriam por semana, bem como a existência ou não de publicações advindas do programa ou projeto. A quinta e última fase compreendia na realização de uma busca aprofundada a fim de mapear possíveis programas e publicações que ainda não haviam sido identificados. Após obter conhecimento sobre os projetos através dos coordenadores e bolsistas previamente contatados, realizou-se uma busca em dados eletrônicos, de forma on-line, no que tange aos sites de cada universidade e de suas respectivas Pró-Reitorias de Extensão (PROEX) e aos sites de ONG's 
e de prefeituras de cada município que possuíam uma universidade pública como parceira em programas desse tipo. Além disso, foram também identificados sites que reúnem projetos e programas nacionais de extensão atuais e antigos, grupos de pesquisas do $\mathrm{CNPq}$, currículo Lattes e redes sociais.

No que se elucida a existência de publicações científicas, houve vários processos. Primeiramente, quando era encontrado um programa que envolvia caminhada, foi realizado um contato por telefone com o coordenador do mesmo, a fim de obter as informações adicionais necessárias, através de um roteiro, referentes ao ano de início do projeto, número de participantes e público-alvo, bem como confirmar ou adicionar informações no que se refere a existência de produção bibliográfica decorrente do programa em questão. Quando positiva a resposta, obtinha-se as referências com o mesmo ou seus respectivos bolsistas, mestrandos e doutorandos, tanto através do contato por telefone quanto por e-mail. Ainda assim, quando a resposta era negativa, na tentativa de ter certeza da não realização de nenhuma publicação, fez-se uma consulta no Currículo Lattes do coordenador, no site da universidade, no site do programa em questão, nos bancos de dados SciELO, LILACS, Google e DeCS e em anais de congresso. Foi considerado publicações realizadas nos últimos 20 anos, de janeiro de 2000 a novembro de 2013, utilizando conjuntamente os termos "programa", "projeto", "caminhada", "atividade física", "saúde", "universidade federal" e "universidade estadual".

Além disso, de modo a garantir o maior número de informações possíveis, foram analisados dissertações, teses e textos encontrados em base de dados e revistas online relacionadas à caminhada orientada dentro dos sites das universidades, quando tal assunto era disponibilizado.

Os dados foram organizados em quadros gráficos, tabelas e mapas para melhor explanação dos resultados e para posterior discussão e conclusão.

Esse projeto foi aprovado pelo Comitê de Ética da Universidade Federal de São Carlos, número do protocolo: 384852 .

\section{RESULTADOS}

A tabela 1 apresenta as universidades públicas que possuem algum Programa de atividade física que envolve a caminhada para a população. Das 99 universidades públicas brasileiras, duas estão em construção e iniciarão seu funcionamento apenas no primeiro semestre de 2014. E ainda, não foi possível o contato com 11 universidades. Desta forma, a busca foi feita em um total de 86 universidades estaduais e federais, e deste total, constatou-se que 54 universidades públicas oferecem algum programa de atividade física envolvendo caminhada.

Dentre estas 54 universidades, a maioria $(n=33)$ apresentou a existência de apenas um programa de caminhada orientada. Do restante, 11 universidades apresentaram dois programas de caminhada e 10 universidades apresentaram a existência de três ou mais programas envolvendo caminhada. Os programas de atividade física que não continham ou não eram exclusivos de caminhada orientada foram excluídos.

Foram encontrados dentre as universidades, 103 programas que envolvem caminhada orientada, sendo que 6 deles eram relacionados somente à pesquisa, e não à extensão. Percebe-se ainda, uma variedade quanto ao público-alvo, 
TABELA 1 - Universidades públicas que possuem Programa de Caminhada Orientada. Abril à Novembro de 2013.

\begin{tabular}{|c|c|c|c|c|c|}
\hline & Estados & $\begin{array}{l}\text { Universidades } \\
\text { que possuem } \\
\text { C.0 }\end{array}$ & $\begin{array}{l}\text { Número de } \\
\text { Programas } \\
\text { Existentes }\end{array}$ & Total & $\begin{array}{l}\text { Características } \\
\text { dos Programas }\end{array}$ \\
\hline & ALAGOAS & $\begin{array}{l}\text { UNEAL } \\
\text { UFAL }\end{array}$ & $\begin{array}{l}1 \\
1\end{array}$ & 2 & $\begin{array}{l}\text { Envolve C.O } \\
\text { Específico de C.O }\end{array}$ \\
\hline ra & AMAZONAS & $\begin{array}{l}\text { UEA } \\
\text { UFAM }\end{array}$ & $\begin{array}{l}1 \\
2\end{array}$ & 3 & $\begin{array}{l}\text { Específico de C.O } \\
\text { Envolve C.O }\end{array}$ \\
\hline$\Delta$ & BAHIA & $\begin{array}{l}\text { UNEB } \\
\text { UEFS } \\
\text { UESB } \\
\text { UFRB }\end{array}$ & $\begin{array}{l}1 \\
3 \\
1 \\
1\end{array}$ & 6 & $\begin{array}{l}\text { Envolve C.O } \\
\text { Envolve C.O } \\
\text { Envolve C.O } \\
\text { Específico de C.O }\end{array}$ \\
\hline 0 & CEARÁ & $\begin{array}{l}\text { UVA } \\
\text { URCA }\end{array}$ & $\begin{array}{l}1 \\
1\end{array}$ & 2 & $\begin{array}{l}\text { Envolve C.0 } \\
\text { Envolve C.0 }\end{array}$ \\
\hline 3 & ESPIRITO SANTO & UFES & 1 & 1 & Envolve C.O \\
\hline 줄 & GOIÁS & $\begin{array}{l}\text { UFG } \\
\text { UEG }\end{array}$ & $\begin{array}{l}3 \\
1\end{array}$ & 4 & $\begin{array}{l}\text { Envolve C.0 } \\
\text { Envolve C.0 }\end{array}$ \\
\hline 嵦 & MARANHÃO & UEMA & 1 & 1 & Envolve C.O \\
\hline 8 & MATO GROSSO & UFMT & 3 & 3 & 2 Específico de C.O \\
\hline 7. & MATO GROSSO DO SUL & UFMS & 2 & 2 & Envolve C.O \\
\hline$\Delta$ & MINAS GERAIS & $\begin{array}{l}\text { UEMG } \\
\text { Unimontes } \\
\text { UFV } \\
\text { UNIFAL } \\
\text { UFJF } \\
\text { UFLA } \\
\text { UFMG } \\
\text { UFOP }\end{array}$ & $\begin{array}{l}1 \\
1 \\
5 \\
2 \\
1 \\
1 \\
1 \\
1 \\
3\end{array}$ & 15 & $\begin{array}{l}\text { Específico de C.O } \\
\text { Específico de C.O } \\
\text { Envolve C.O } \\
\text { Envolve C.O } \\
\text { Específico de C.O } \\
\text { Envolve C.O } \\
\text { Envolve C.O } \\
\text { Envolve C.O }\end{array}$ \\
\hline 2 & PARANÁ & $\begin{array}{l}\text { UEL } \\
\text { UEPG } \\
\text { UNICENTRO } \\
\text { UNIOESTE } \\
\text { UFPR } \\
\text { UTFPR }\end{array}$ & $\begin{array}{l}2 \\
2 \\
2 \\
1 \\
1 \\
1\end{array}$ & 9 & $\begin{array}{l}01 \text { Específico de C.O } \\
\text { Específico de C.O } \\
\text { Envolve C.O } \\
\text { Envolve C.0 } \\
\text { Envolve C.O } \\
\text { Envolve C.O }\end{array}$ \\
\hline & PARAÍBA & $\begin{array}{l}\text { UFPB } \\
\text { UEPB }\end{array}$ & $\begin{array}{l}1 \\
1\end{array}$ & 2 & $\begin{array}{l}\text { Envolve C.0 } \\
\text { Envolve C.0 }\end{array}$ \\
\hline$\infty$ & PERNAMBUCO & UPE & 2 & 2 & Envolve C.O \\
\hline 垔 & PIAUÍ & UESPI & 1 & 1 & Envolve C.O \\
\hline d & RIO DE JANEIRO & $\begin{array}{l}\text { UERJ } \\
\text { UFF } \\
\text { UFRJ } \\
\text { UNIRIO }\end{array}$ & $\begin{array}{l}1 \\
2 \\
1 \\
1\end{array}$ & 5 & $\begin{array}{l}\text { Envolve C.O } \\
\text { Envolve C.O } \\
\text { Envolve C.0 } \\
\text { Envolve C.0 }\end{array}$ \\
\hline $\bar{x}$ & RIO GRANDE DO NORTE & $\begin{array}{l}\text { UERN } \\
\text { UFERSA } \\
\text { UFRN }\end{array}$ & $\begin{array}{l}1 \\
1 \\
1\end{array}$ & 3 & $\begin{array}{l}\text { Envolve C.O } \\
\text { Envolve C.O } \\
\text { Específico de C.O }\end{array}$ \\
\hline (i) & RIO GRANDE DO SUL & $\begin{array}{l}\text { UFPel } \\
\text { UFSM } \\
\text { UNIPAMPA } \\
\text { UFRGS } \\
\text { UFCSPA }\end{array}$ & $\begin{array}{l}4 \\
3 \\
1 \\
2 \\
1\end{array}$ & 11 & $\begin{array}{l}\text { Envolve C.0 } \\
\text { Envolve C.0 } \\
\text { Envolve C.O } \\
01 \text { Específico de C.O } \\
\text { Envolve C.0 }\end{array}$ \\
\hline 4 & RORAÍMA & UERR & 1 & 1 & Envolve C.0 \\
\hline 20 & SANTA CATARINA & $\begin{array}{l}\text { UFSC } \\
\text { UDESC }\end{array}$ & $\begin{array}{l}6 \\
6\end{array}$ & 12 & $\begin{array}{l}\text { Envolve C.0 } \\
03 \text { Envolve C.O }\end{array}$ \\
\hline 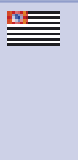 & SÃO PAULO & $\begin{array}{l}\text { USP } \\
\text { UNICAMP } \\
\text { UNESP } \\
\text { UFSCAR } \\
\text { UNIFESP }\end{array}$ & $\begin{array}{l}14 \\
1 \\
2 \\
1 \\
1\end{array}$ & 19 & $\begin{array}{l}12 \text { Envolvem de C.0 } \\
\text { Envolve C.O } \\
\text { Envolve C.O } \\
\text { Específico de C.O } \\
\text { Envolve C.O }\end{array}$ \\
\hline
\end{tabular}


cabendo informar os maiores resultados, onde, do total dos programas, $34,9 \%$ $(n=36)$ eram destinados aos idosos, 25,2\% $(n=26)$ para o público externo às universidades, $22,3 \%(\mathrm{n}=23)$ ao público interno das universidades - técnicos, servidores, docentes e discentes - e $11,6 \%(n=12)$ não foi possível obter essa informação. Cabe informar que ainda há uma minoria dos programas destinados a outras populações distintas $(n=6)$.

A elaboração de programas que envolvem caminhada esteve associada à existência de cursos da área da saúde naquela universidade em questão, visto que, normalmente, tais programas foram implantados e eram coordenados por professores de cursos como Educação Física, Gerontologia, Fisioterapia e Enfermagem.

Esses programas variam quanto ao tempo de implantação dos mesmos nas universidades, sendo o mais antigo identificado, com início em 1984, da Universidade Federal de Santa Maria (UFSM) e o mais atual encontrado, foi na Universidade Federal de São Carlos (UFSCar), que teve início em outubro de 2013. Vale ressaltar que o ano de 2013, foi na média onde houve o maior número de implantação de programas nessas universidades.

Houve universidades que implantaram mais de um programa de caminhada orientada. Dentre elas, destaca-se a Universidade de São Paulo (USP) com doze programas de caminhada orientada, sendo que o ano de implantação varia de 1994 à 2013. Como forma de ilustrar, é possível mencionar a Universidade do Estado de Santa Catarina (UDESC) com seis programas implantados entre os anos de 1991 e 2013, bem como a Universidade Federal de Santa Catarina (UFSC), também com seis programas de caminhada implantados, entre 1985 e 2009

A tabela 2 refere-se ao número de publicações na literatura das universidades federais e estaduais que possuem um programa de atividade física envolvendo caminhada. Foram encontrados programas implantados desenvolvidos no período de 1984 a 2013 (data não pré-determinada). Das 54 universidades as quais oferecem programas de caminhada orientada, 30 realizaram alguma publicação científica a respeito da temática. Destas que realizaram publicação, pôde-se observar que a quantidade foi elevada na maioria, sendo no mínimo uma e variando até a máxima de 104 publicações existentes. No total, foram 344 publicações, tendo um destaque o estado de SP ( $n=154)$, SC $(n=58)$ e RS ( $n=35)$.

A figura 2 é referente ao número de programas de atividade física envolvendo caminhada nos estados brasileiros. Um maior destaque das universidades que implantaram esses programas está no estado de São Paulo, totalizando 19 programas concentrados em cinco grandes universidades (USP, UNESP, UFSCAR, UNIFESP e UNICAMP).

$\mathrm{O}$ estado de Minas Gerais possui 15 programas dentre as universidades federais e estaduais (UEMG, Unimontes, UFV, UNIFAL, UFJF, UFLA, UFMG e UFOP). E ainda, Rio Grande do Sul, onde contém 11 programas, centrados em cinco universidades (UFPel, UFSM, UNIPAMPA, UFRGS e UFCSPA) e no estado de Santa Catarina (UFSC e UDESC), com também 11 programas.

É possível, portanto, observar que muitas universidades possuem mais de um programa, o que totaliza maior quantidade no estado, de modo geral.

A figura 3 se refere ao número de publicações existentes dos programas que encontramos nos estados brasileiros. É possível observar que o maior número dessas publicações, concentram-se nos estados da região sul e sudeste. 
Destacando-se o estado de São Paulo, o qual totaliza 154 publicações referentes aos programas de extensão que envolvem caminhada. Além dos estados de Santa Catarina e Rio Grande do Sul, que chega a um número de 56 e 35 publicações respectivamente.

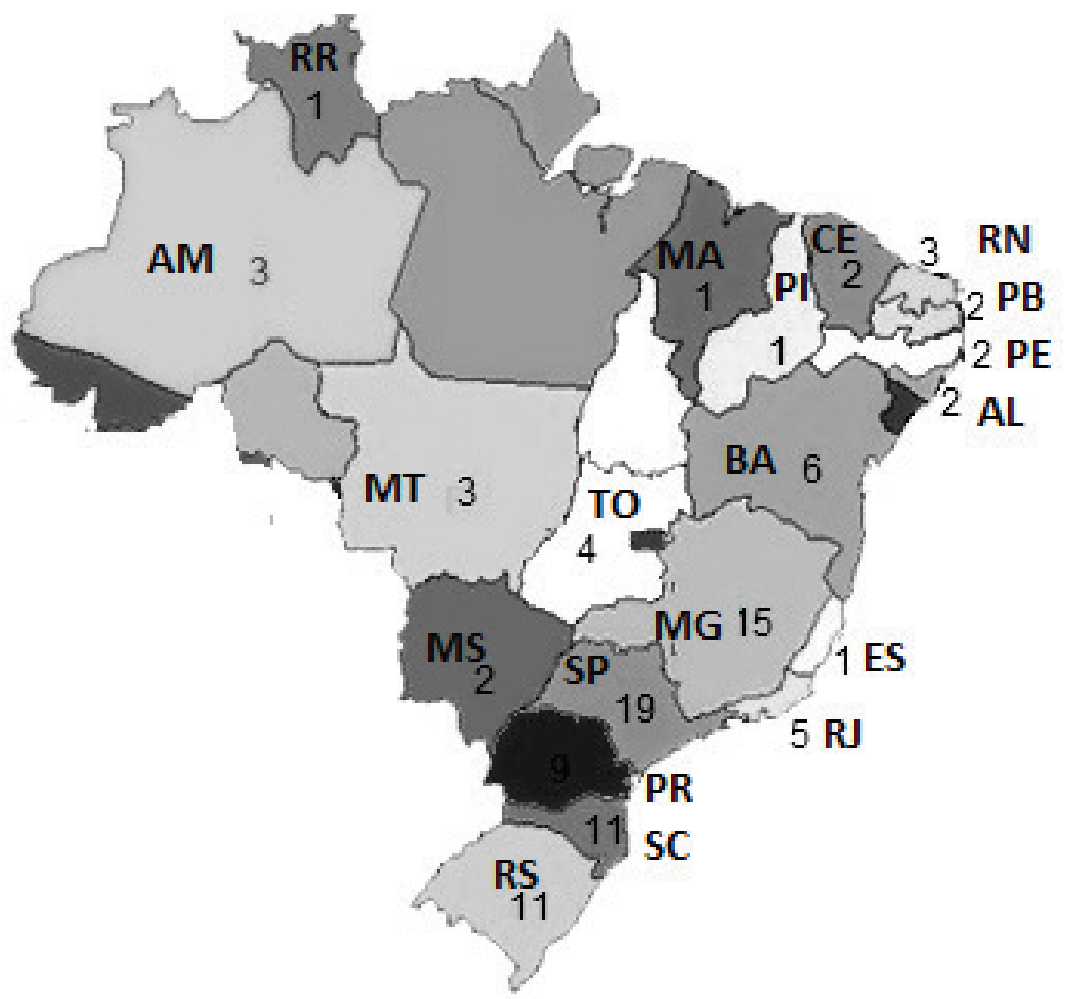

FIGURA 2 - Número de programas de Caminhada Orientada em universidades públicas nos estados brasileiros. Abril à Novembro de 2013.

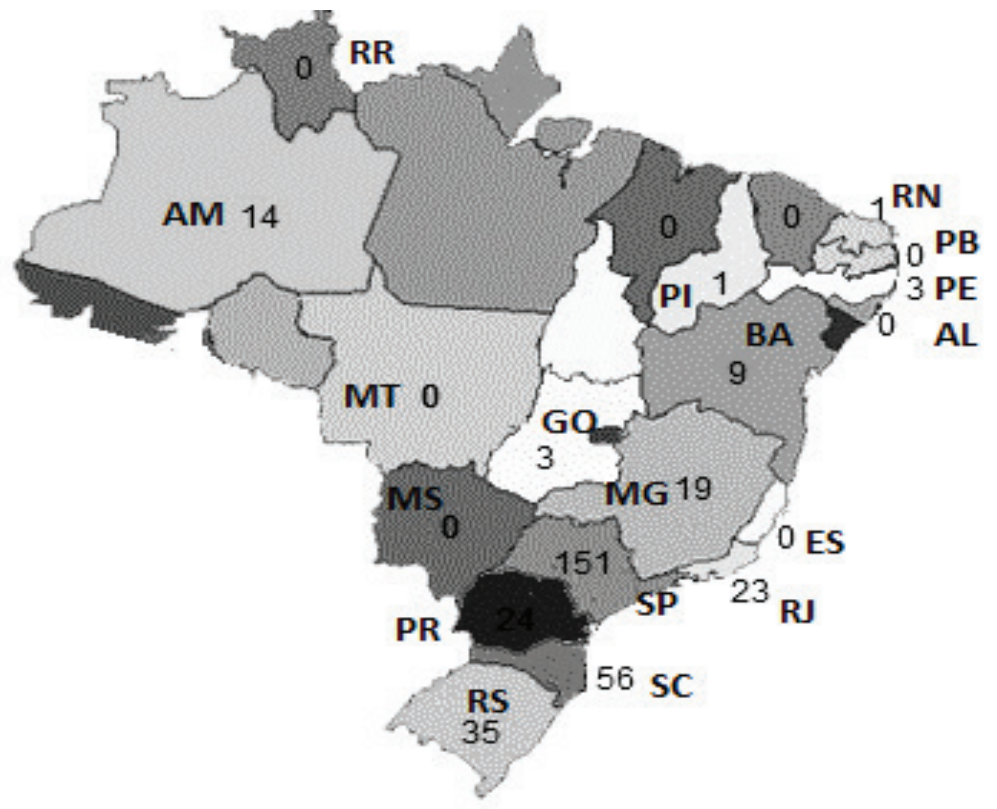

FIGURA 3 - Número de publicações existentes dos programas de atividade física envolvendo caminhada em universidades públicas nos estados brasileiros. Abril à Novembro de 2013. 
TABELA 2 - Número de publicações dos programas atividade física envolvendo caminhada em universidades públicas. Abril à Novembro de 2013.

\begin{tabular}{|c|c|c|c|c|}
\hline & Estados & $\begin{array}{l}\text { Universidades } \\
\text { que possuem } \\
\text { C.0 }\end{array}$ & $\begin{array}{l}\text { Número de } \\
\text { Publicações } \\
\text { Existentes }\end{array}$ & Total \\
\hline 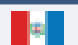 & ALAGOAS & UNEAL & 0 & 0 \\
\hline ab & AMAZONAS & $\begin{array}{l}\text { UEA } \\
\text { UFAM }\end{array}$ & $\begin{array}{l}1 \\
13\end{array}$ & 14 \\
\hline$\Delta$ & BAHIA & $\begin{array}{l}\text { UNEB } \\
\text { UEFS } \\
\text { UESB } \\
\text { UFRB }\end{array}$ & $\begin{array}{l}2 \\
7 \\
0 \\
0\end{array}$ & 9 \\
\hline 0 & CEARÁ & $\begin{array}{l}\text { UVA } \\
\text { URCA }\end{array}$ & $\begin{array}{l}0 \\
0\end{array}$ & 0 \\
\hline$=$ & ESPIRITO SANTO & UFES & 0 & 0 \\
\hline 晒= & GOIÁS & $\begin{array}{l}\text { UFG } \\
\text { UEG }\end{array}$ & $\begin{array}{l}3 \\
0\end{array}$ & $\begin{array}{l}3 \\
0\end{array}$ \\
\hline$\underline{\underline{\underline{\underline{\underline{W}}}}}$ & MARANHÃO & UEMA & 0 & 0 \\
\hline & MATO GROSSO & UFMT & 0 & 0 \\
\hline 7. & MATO GROSSO DO SUL & UFMS & 0 & 0 \\
\hline 4 & MINAS GERAIS & $\begin{array}{l}\text { UEMG } \\
\text { Unimontes } \\
\text { UFV } \\
\text { UNIFAL } \\
\text { UFJF } \\
\text { UFLA } \\
\text { UFMG } \\
\text { UFOP }\end{array}$ & $\begin{array}{l}0 \\
3 \\
3 \\
0 \\
9 \\
3 \\
0 \\
1\end{array}$ & 19 \\
\hline 2 & PARANÁ & $\begin{array}{l}\text { UEL } \\
\text { UEPG } \\
\text { UNICENTRO } \\
\text { UNIOESTE } \\
\text { UFPR } \\
\text { UTFPR }\end{array}$ & $\begin{array}{l}0 \\
3 \\
6 \\
13 \\
1 \\
1\end{array}$ & 24 \\
\hline . & PARAÍBA & $\begin{array}{l}\text { UFPB } \\
\text { UEPB }\end{array}$ & $\begin{array}{l}0 \\
0\end{array}$ & 0 \\
\hline$\infty$ & PERNAMBUCO & UPE & 3 & 3 \\
\hline 믈 & PIAUÍ & UESPI & 1 & 1 \\
\hline d & RIO DE JANEIRO & $\begin{array}{l}\text { UERJ } \\
\text { UFF } \\
\text { UFRJ } \\
\text { UNIRIO }\end{array}$ & $\begin{array}{l}1 \\
15 \\
0 \\
7\end{array}$ & 23 \\
\hline w & RIO GRANDE DO NORTE & $\begin{array}{l}\text { UERN } \\
\text { UFERSA }\end{array}$ & $\begin{array}{l}1 \\
0\end{array}$ & 1 \\
\hline (a) & RIO GRANDE DO SUL & $\begin{array}{l}\text { UFPel } \\
\text { UFSM } \\
\text { UNIPAMPA } \\
\text { UFRGS } \\
\text { UFCSPA }\end{array}$ & $\begin{array}{l}9 \\
20 \\
1 \\
5 \\
0\end{array}$ & 35 \\
\hline 21 & RORAÍMA & UERR & 0 & 0 \\
\hline 30: & SANTA CATARINA & $\begin{array}{l}\text { UFSC } \\
\text { UDESC }\end{array}$ & $\begin{array}{l}24 \\
34\end{array}$ & 58 \\
\hline 柴 & SÃO PAULO & $\begin{array}{l}\text { USP } \\
\text { UNICAMP } \\
\text { UNESP } \\
\text { UFSCAR } \\
\text { UNIFESP }\end{array}$ & $\begin{array}{l}105 \\
32 \\
16 \\
1 \\
0\end{array}$ & 154 \\
\hline
\end{tabular}




\section{DISCUSSÃO}

A pesquisa ilustra que o Brasil vem desenvolvendo de forma significativa práticas de promoção de saúde através do oferecimento de atividade física em universidades públicas. Os resultados mostram que as Universidades Federais e Estaduais brasileiras tem se atentado em relação à promoção de qualidade de vida e saúde para a população através da promoção de atividade física. Das Universidades contatadas, quase dois terços possuiu ou possui algum tipo de programa relacionado à prática da caminhada como forma de atividade física $\mathrm{e}$ publicações referentes à mesma. Isso mostra que esses locais são privilegiados com o oferecimento de acesso a programas de caminhada na universidade para a população. Isso pode ter ocorrido devido a maior conscientização quanto à importância da atividade física para a saúde da população em conjunto com as condições estruturais e o interesse das Universidades em desenvolver esse tipo de atividade para a comunidade.

Além disso, conforme tem sido preconizado nas políticas públicas, a promoção da saúde tem como objetivo promover a qualidade de vida e reduzir vulnerabilidade e riscos à saúde relacionados aos seus determinantes e condicionantes - modos de viver, condições de trabalho, habitação, ambiente, educação, lazer, cultura, acesso a bens e serviços essenciais. Esta responsabilidade é compartilhada, do Estado (políticas públicas saudáveis), dos indivíduos (desenvolvimento de habilidades pessoais), do sistema de saúde (reorientação do sistema de saúde) e de parcerias intersetoriais ${ }^{19}$. Portanto, os dados desse estudo mostraram que grande parte das universidades públicas tem atendido às recomendações de serem mais um espaço de responsabilidade do Estado para o oferecimento de programas de atividade física.

Os programas oferecidos nas Universidades variam o público alvo, sendo a maioria desses idosos, públicos externos às universidades, internos das universidades, e uma minoria voltada para o público maior de 30 anos, adolescentes, obesos, pessoas com doença de Parkinson, fibromialgia, deficientes, moradores de um asilo, usuários de uma Unidade Básica de Saúde (UBS), cardíacos, pessoas independentes e saudáveis, porém, importante destacar que o objetivo entre eles é comum, de promoção da saúde.

Os dados dessa pesquisa demonstraram a utilização da atividade física como ferramenta de cuidados de saúde da população como um todo, visto que, nas últimas décadas, com o processo de industrialização, urbanização e mecanização, houve significativas mudanças no padrão de morbimortalidade mundial, verificando que a falta de atividades físicas regulares vem sendo considerada um fator de risco primário e de muitos agravos à saúde ${ }^{20}$. Portanto, a implantação de programas deste gênero tem sido muito importante para população e corrobora com a preocupação das organizações em incluírem a atividade física no contexto de suas atividades e serviços oferecidos ${ }^{21}$.

Durante a busca quanto aos programas, foi possível perceber que algumas Universidades, apesar de não oferecerem a prática de atividades físicas orientadas, possuem núcleos de pesquisa sobre a atividade física, o que pode ainda futuramente desenvolver mais programas de atividade física para a comunidade.

Alguns estados destacaram-se quanto à concentração de programas existentes que envolvem caminhada e à quantidade de publicações, sendo eles São Paulo, Minas Gerais, Santa Catarina e Rio Grande de Sul. Esse fato pode 
ser justificado por algumas características que são destaques nos municípios e estados dessas regiões. Primeiramente podemos destacar que muitos destes locais apresentam os maiores IDH-M (Índice de Desenvolvimento Humano - Municipal) do Brasil, pelo maior nível de enriquecimento destes, pela maior qualidade de vida da população e longevidade e por elevados níveis de educação e qualidade de ensino ${ }^{22}$.

Além disso, os dados do IFDM (Índice Firjan de Desenvolvimento Municipal) de 2012 corroboram esses dados, pois São Paulo encontra-se em $1^{\circ}$ lugar no ranking entre os estados brasileiros, com 0,89; Santa Catarina, em $3^{\circ}$ lugar, com índice de 0,82 ; Minas Gerais, em $5^{\circ}$ lugar com 0,81e Rio Grande do Sul, em $6^{\circ}$ lugar com $0,81^{23}$. Esse índice avalia o desenvolvimento, segundo variáveis de educação, saúde e renda e emprego.

Tềm-se ainda, que os maiores números de programas, bem como publicações a respeito, também podem ser explicados pelo fato da concentração de universidades, com melhores pontuações, de acordo com o Índice Geral de Cursos (IGC) da INEP (Instituto Nacional de Estudos e Pesquisas), que é um indicador de qualidade de instituições de educação superior, que considera em sua composição, a qualidade dos cursos de graduação e de pós-graduação (mestrado e doutorado) ${ }^{24}$.

Nesse aspecto, segundo divulgações do MEC, as universidades concentradas na região sul e sudeste do país, estão classificadas entre as dez melhores universidades do Brasil ${ }^{25}$. Desse modo, esse indicativo pode estar associado a promoção da melhoria da qualidade e pertinência das atividades desenvolvidas, bem como em compromissos científicos e sociais, onde podem estar implícitos a promoção de programas como de caminhada orientada à população.

Portanto, conforme corroboram os dados, os melhores índices de desenvolvimento dos estados podem ser fatores propulsores para se constituírem melhores universidades e parcerias com órgãos públicos e privados nos municípios e, por conseguinte, maiores estímulos e ações que partem dessas universidades, voltadas à população através de projetos de extensão e pesquisa.

Especificamente os projetos de extensão possuem um papel fundamental de aplicar o conhecimento adquirido com a população. É uma forma de interação que deve existir entre a universidade e a comunidade, sendo uma espécie de ponte permanente entre a universidade e os diversos setores da sociedade, de modo a funcionar como uma via de mão dupla, em que a Universidade leva conhecimentos e/ou assistência à comunidade, e recebe dela suas reais necessidades, seus anseios, aspirações e também aprendizado de seus valores e cultura ${ }^{26}$.

No caso de um programa de atividade física oferecido em universidades, em especial aqueles desenvolvidos enquanto projetos de pesquisa e extensão em conjunto, possibilita aprofundar os conhecimentos existentes, tanto no que se refere a instruções atualizadas sobre os benefícios e recomendações de atividade física quanto na formação profissional de estudantes de graduação e pós graduação. Neste aspecto cabe destacar os desafios e limitações apresentados pelos docentes das universidades da área de intervenções com atividade física na realização de forma associada entre pesquisa e extensão, principalmente em estudos experimentais, os quais envolvem aleatorização e controle rigoroso de variáveis.

No que se refere aos benefícios da quantidade de programas existentes no 
país especificamente para os usuários, estes colaboram para a prevenção e controle de doenças como hipertensão, Diabetes Mellitus e doenças cardiovasculares, melhoram o condicionamento físico geral, humor, aspectos cognitivos, previnem contra a obesidade e promovem interação social ${ }^{27,28}$. Vale ressaltar a importância de estimular e investir na criação de mais programas de caminhada orientada como forma de atividade física, pois se trata de uma alternativa de promoção da saúde de baixo custo e que apresenta melhorias significantes para a qualidade de vida das pessoas que aderem ao programa.

Também foi possível perceber a partir dos resultados que grande parte dos programas tem como público alvo os idosos, fato que, como afirma $\mathrm{Nadai}^{29}$, é de extrema relevância, pois pré-concepção de envelhecimento como algo ruim e desagradável, torna tudo mais difícil, conduzindo o idoso ao isolamento, e, tudo que produziu, criou, viveu e amou parecem perder o sentido. A atividade física dá a oportunidade de resgatar esses sentimentos, auxiliando na descoberta de uma nova perspectiva de vida e de reinserção à sociedade ${ }^{30}$.

Freitas realizou um estudo referente a motivos de adesão dos idosos a programas de atividade física. Os motivos mais importantes relatados pelos participantes foram: melhorar a saúde $(84,2 \%)$; melhorar o desempenho físico (70,8\%); adotar estilo de vida saudável $(62,5 \%)$; reduzir o estresse $(60,8 \%)$; acatar prescrição médica (56,7\%); auxiliar na recuperação de lesões $(55 \%)$; melhorar a auto-imagem (50,8\%); melhorar a auto-estima e relaxar $(47,5 \%)^{31}$. De acordo com os dados dessa pesquisa, as universidades públicas tem sido, portanto, um local para que os idosos consigam alcançar esses objetivos.

O estudo realizado apresentou limitações quanto à obtenção de informações sobre a existência e características dos programas oferecidos, por conta de dificuldades em contatar os docentes responsáveis pelos programas de extensão encontrados. Em alguns poucos casos estes não responderam aos e-mails ou telefonemas, o que pode ter limitado a coleta de dados. Outra dificuldade percebida pelos pesquisadores foi quanto às universidades ainda em construção, pois não foi possível entrar em contato com as mesmas. Por fim, uma limitação do estudo foi não questionar o período de existência de cada programa ou projeto nas universidades.

Com base nos resultados apresentados acima podemos perceber que o número de programas envolvendo caminhada é amplo. O que se pode concluir é que as universidades públicas vêm se atentando à importância do desenvolvimento de programas de atividade física orientada. Esses programas se apresentam como uma alternativa para uma possível melhoria da qualidade de vida e da promoção do bem-estar, promovendo acesso da população à prática de atividade física também por meio dos serviços das universidades públicas.

Vale ressaltar que o foco do estudo se deu apenas em Universidades públicas, pois em grande parte das instituições privadas não existe, de fato, liberdade acadêmica suficiente para os docentes no que se refere ao ensino, extensão e pesquisa, pois a criação de cursos, o currículo e até as ementas das disciplinas oferecidas são controladas pela mantenedora, do mesmo modo que as raras pesquisas existentes ${ }^{29}$. Deste modo, considerou-se que seria mais válido focar a busca apenas em Universidades públicas, pois existe maior autonomia para explorar a questão da tríade ensino-pesquisa-extensão. Sugerem-se futuros estudos para verificar a realidade da existência de programas de atividade física nesse contexto. 
Os dados dessa pesquisa demonstraram a existência expressiva de programas de caminhada em universidades públicas, fortalecendo dessa forma a reflexão sobre o papel das universidades públicas no âmbito da pesquisa e extensão na área de atividade física e saúde. Trata-se, portanto, de consolidar a divulgação da atuação das universidades na promoção da saúde da população, em especial na forma de publicações científicas. É possível ampliar ainda mais a respeito da interação entre comunidade interna e externa. Destacase nesse sentido a importância de estudos envolvendo a prática da extensão universitária para que o conhecimento possa se tornar tangível a todos.

\section{REFERÊNCIAS}

1. Hallal PC, Carvalho YM, Tassitano RM, Tenório MCM, Warschauer M, Reis RS, et al. Avaliação do programa de promoção da atividade física Academia da Cidade de Recife, Pernambuco, Brasil: percepções de usuários e não-usuários. Cad. Saúde Pública. 2010; 26(1): 70-78.

2. Rütten A, Abu-Omar K, Gelius P, Schow D. Physical inactivity as a policy problem: applying a concept from policy analysis to a public health issue. Health Res Pol Syst. 2013; 11: 9 .

3. Garber CE, Blissmer B, Deschenes MR, Franklin BA, Lamonte MJ, Lee IM, et al. Quantity and quality of exercise for developing and maintaining cardiorespiratory, musculoskeletal, and neuromotor fitness in apparently healthy adults: guidance for prescribing exercise. American College of Sports Medicine position stand. Med Sci Sports Exerc. 2011; 43 (7): 1334.

4. Hallal PC, Andersen LB, Bull FC, Guthold R, Haskell W, Ekelund U. Global physical activity levels: surveillance progress, pitfalls, and prospects. The Lancet. 2012; 380: 247-257.

5. Brasil. Ministério da Saúde. Vigitel Brasil 2012: Vigilância de fatores de risco e proteção para doenças crônicas por inquérito telefônico. Brasília; 2012.

6. Schmidt MI, Duncan BB, Silva GA, Menezes AM, Monteiro CA, Barreto SM, et al.Doenças crônicas não transmissíveis no Brasil: carga e desafios atuais. The Lancet. 2011; 4: 61-74.

7. Moretti AC, Almeida V, Westphal MF, Bógus CM..Práticas Corporais/Atividade Física e Políticas Públicas de Promoção da Saúde. Saúde Soc. São Paulo. 2009; 18(2): 346-54.

8. Bielemann RM, Knuth AG, Hallal PC. Atividade física e redução de custos por doenças crônicas ao sistema Único de Saúde. RevBras de AtivFís Saúde. 2010; 15(1).

9. Silveira MM, Pasqualotti A, Colussi EL. Prevalência de doenças crônicas e prática de atividade física em adultos e idosos. Rev Bras Prom Saúde. Fortaleza. 2012; 25(2): 209-

10. Amorim C, Knuth A, Cruz D, Malta D, Reis R, Hallal P. Description of the physical activity promotion programs funded by the Brazilian Ministry of Health. RevBrasAtivFis Saúde. Pelotas/RS. 2013; 18(1):63-74.

11. Krug RR, Marchesan M, Conceição JCR, Mazo GZ, Antunes GA, Romitti JC. Contribuições da caminhada como atividade física de lazer para idosos. Licere. Belo Horizonte. 2011; 14(4).

12. Nunes MES, Santos S. Avaliação funcional de idosos em três programas de atividade física: caminhada, hidroginástica e Lian Gong. RevPortCiên Desp. 2009; 9(2-3): 150-59.

13. Freitas RPA, Medeiros ACQ, Cavalcante ARS, Souza DE. (Re)fazendo a caminhada: otimizando uma estratégia de controle da pressão arterial. Extensão e Sociedade. 2010; 1(2).

14. Florindo AA, Mielke GI, Gomes GAO, Ramos LR, Bracco MM, Parra DC, et al . Physical activity counseling in primary health care in Brazil: a national study on prevalence and associated factors. BMC Public Health. 2013; 13: 1-10.

15. Carvalho SMS. Reflexões sobre a extensão na universidade pública brasileira. RevParticipAção. Brasília. 2009; (16): 12-20.

16. 16.Gobbi, S. Programa de atividade física para idosos: Integração comunidade universidade. RevCiênc Ext. 2008; 4: 86-93. 
17. Nakamura PM, Papini CB, Chiyoda A, Gomes GAO, Netto Ava, Teixeira IP, et al. Programa de intervenção para a prática de atividade física: Saúde Ativa Rio Claro. RevBrasAtivFís Saúde. 2010; 15(2): 128-32.

18. Barros CR, Cezaretto A, Salvador EP, Santos TC, Siqueira-Catania A; Ferreira SRG. Implementação de programa estruturado de hábitos de vida saudáveis para redução de risco cardiometabólico. ArqBrasEndocrinolMetab. 2013; 57(1): 7-18.

19. Política nacional de atenção básica. Série Pactos pela saúde Brasília: Ministério da Saúde. Secretaria de Atenção à Saúde. Departamento de Atenção Básica. 42006.

20. Brasil. Ministério da Saúde. A vigilância, o controle e a prevenção das doenças nãotransmissíveis: DCNT no contexto do Sistema único de Saúde Brasileiro. Brasília; 2005.

21. Hallal PC, Dumith SC, Bastos JP, Reichert FF, Siqueira FV, Azevedo MR. Evolução da pesquisa epidemiológica em atividade física no Brasil: revisão sistemática. RevSaude Publica. 2007; 41(3): 453-60.

22. PNUD. Programa das Nações Unidas para o Desenvolvimento. IDH-M. Índice de Desenvolvimento Humano-Municipal. Disponível em: http://www.pnud.org.br/atlas/ ranking/Ranking-IDHM-Municipios-2010.aspx. Acesso em: 08/07/2013.

23. IFDM. Índice FIRJAN de Desenvolvimento Municipal. Consulta ao índice. Disponível em: http://www.firjan.org.br/ifdm/consulta-ao-indice/. Acesso em: 16/07/2013.

24. Brasil. Ministério da Educação. Instituto Nacional de Estudos e Pesquisas Educacionais Anísio Teixeira (INEP). Brasília; 2011.

25. Brasil. Ministério da Educação. MEC. Disponível em: www.mec.gov.br/囚. Acesso em: 19/07/2013.

26. Silva MG. Universidade e sociedade: cenário da extensão universitária? Disponível em: < http://www.anped11.uerj.br/23/1101t.htm> Acessado em <21/01/2014>

27. Garber, C. E., B. Blissmer, et al. American College of Sports Medicine position stand. Quantity and quality of exercise for developing and maintaining cardiorespiratory, musculoskeletal, and neuromotor fitness in apparently healthy 107 adults: guidance for prescribing exercise. Medicine and science in sports and exercise, v.43, n.7, p.1334. 2011.

28. Lee, I. M., E. J. Shiroma, et al. for the Lancet Physical Activity Series Working Group Effect of physical inactivity on major non-communicable diseases worldwide: an analysis of burden of disease and life expectancy. Lancet, p.61031-9. 2012.

29. Nadai, A. Programa de atividades físicas e terceira idade. Motriz. 1995; 1(2): 120-123.

30. 31. Franchi KMB, Junior RMM. Atividade física: uma necessidade para a boa saúde na terceira idade. Revista Brasileira em Promoção da Saúde, vol. 18, núm. 3,pp. 152-156, 2005

31. Freitas CMSM, Santiago MS, Viana AT, Leão AC, Freyre C. Aspectos motivacionais que influenciam a adesão e manutenção de idosos a programas de exercícios físicos. RevBrasCineantropom Desempenho Humano. 2007; 9(1): 92-100.

\section{ENDERECOO PARA}

CORRESPONDÊNCIA

NATÁLIA OIRING DE CASTRO CEZAR

Rua dos Cronópios, n89. Condomínio

Jardim das Flores. São José dos

Campos - SP. Brasil. CEP: 12247-700

Telefone: (16) 98100-8080

E-mail: nataliaoiringlayahoo.com.br 